\title{
SOLAR ENERGY DRIVEN MOSQUITO REPELLER SYSTEM USING ARDUINO UNO
}

\author{
Dr. Dhiraj Sunehra \\ Department of Electronics \& Communication Engineering \\ Faculty of Engineering \& Technology \\ Jawaharlal Nehru Technological University Hyderabad, Hyderabad, India
}

\begin{abstract}
Mosquitoes and other insects are harmful for humans as they can cause many diseases which are harmful for human health. In order to avoid the mosquitoes, people use chemical repellents which affect the environment adversely. Use of mosquito repeller skin creams can cause skin problems. In this paper, a mosquito repeller system that operates by harvesting the solar energy from sun is implemented. The proposed mosquito repeller system consumes low power with an adequate affordability, availability, and versatility. The software tool used is Arduino IDE, which is used to write an embedded code for Arduino board. The designed system consumes very low power and can be effectively used in lawns and environment parks to repel the mosquitoes.
\end{abstract}

Key words: Arduino Uno, Mosquito repeller system, Energy harvesting, Arduino IDE

Cite this Article: Dr. Dhiraj Sunehra, Solar Energy Driven Mosquito Repeller System Using Arduino Uno, International Journal of Advanced Research in Engineering and Technology, 10(2), 2019, pp 472-481.

http://iaeme.com/Home/issue/IJARET?Volume=10\&Issue $=2$

\section{INTRODUCTION}

Mosquitoes are one of the few animals that cause itchy, irritating bites when we wish to relax in our bedrooms, or in the lawns or a garden. The buzz of their wings can be a nuisance factor and can wake a person in deep sleep also. Mosquitoes are carriers of some of the deadliest diseases such as malaria and dengue. Mosquitoes use exhaled carbon dioxide, body odors and temperature, and movement to home in on their victims. Only female mosquitoes have the mouth parts necessary for sucking blood. They use the blood not for their own nourishment but as a source of protein for their eggs. For food, both males and females eat nectar and other plant sugars.

All mosquitoes need water to breed, so eradication and population-control efforts usually involve removal or treatment of standing water sources. Insecticide spraying to kill adult mosquitoes is also widespread. However, global efforts to stop the spread of mosquitoes are having little effect, and many scientists think global warming will likely increase their number and range. Mosquitoes are mostly found in early morning and late evening near areas of standing water, near plants and in homes, offices, etc [1]. 
In this paper, a low-cost mosquito repeller system is developed using Arduino Uno, repeller system and a small solar panel. The solar panel absorbs solar energy of the Sun to charge during the daytime using a rechargeable battery. The harvested energy can be used to turn on and operate the mosquito repeller system during evening and night time. The mosquito repeller system generally employs intermittent and high frequencies to drive away the insects. The moment a mosquito or any bug comes in contact with the meshes, the stored high voltage in the capacitor discharges violently through the body of the entangled bug creating a big spark and electrocuting it instantly.

The Solar Photovoltaic cell uses a technology that converts sunlight into direct current by using semiconductor. When the sunlight hits the semiconductor within the photovoltaic cell, electrons are freed and from an electric current. This technology is very useful for energy harvesting and can be efficiently used to drive wide variety of loads [2].

\section{HARDWARE DESCRIPTION}

\subsection{Block Diagram}

Figure 1 shows the block diagram of a solar energy driven mosquito repeller system. It consists of a power supply unit having a solar panel and a sealed maintenance free (SMF) rechargeable battery which supplies power to the Arduino Uno. The arduino provides necessary power to drive the mosquito repeller system using a relay driver. The real time clock (RTC) module is used to provide necessary timing.

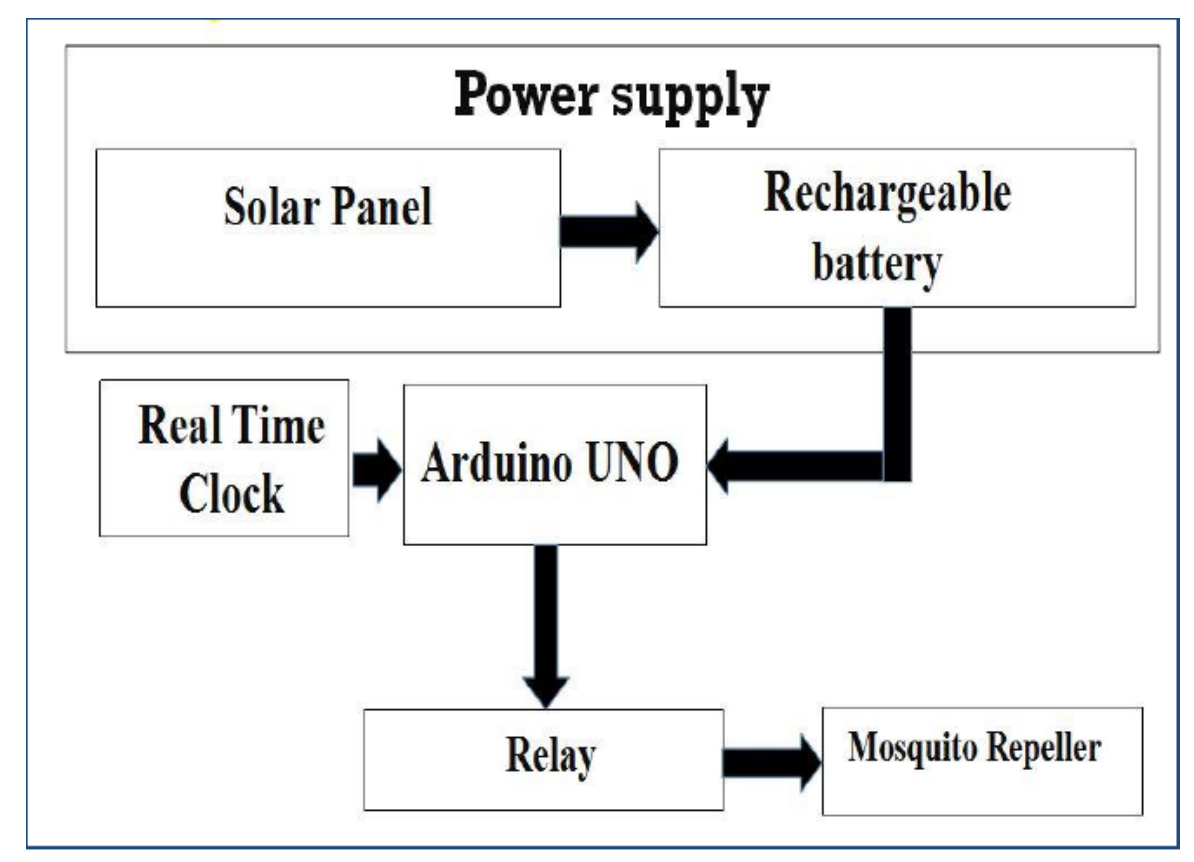

Figure 1 Block Diagram of Solar Energy Driven Mosquito Repeller System

\subsection{Arduino Uno Board}

Arduino Uno is a microcontroller board based on the ATmega328P (Figure 2). It has 14 digital input/output pins (of which 6 can be used as PWM outputs), 6 analog inputs, a $16 \mathrm{MHz}$ quartz crystal, a USB connection, a power jack, an ICSP header and a reset button [3]. It contains everything needed to support the microcontroller; simply connect it to a computer with a USB cable or power it with a AC-to-DC adapter or battery to get started. 


\subsubsection{Salient Features of Arduino Uno}

The salient features of ATmega328P microcontroller are given below:

$>$ Advanced RISC architecture

$>131$ powerful instructions - most single clock cycle execution

$>32 \times 8$ general purpose working registers

$>1$ Kbytes EEPROM

2Kbytes internal SRAM

$>$ High performance, low power AVR ${ }^{\circledR}$ 8-bit microcontroller

$>23$ programmable $\mathrm{I} / \mathrm{O}$ lines

Peripheral features:

$>$ Two 8-bit Timer/Counters with separate prescaler and compare mode

$>$ One 16-bit Timer/Counter with separate prescaler, compare mode, and capture mode

$>$ Real time counter with separate oscillator

$>$ Six PWM channels

Operating voltage:

$>2.7 \mathrm{~V}$ to $5.5 \mathrm{~V}$ for ATmega328P

Temperature range:

Automotive temperature range: $-40^{\circ} \mathrm{C}$ to $+125^{\circ} \mathrm{C}$

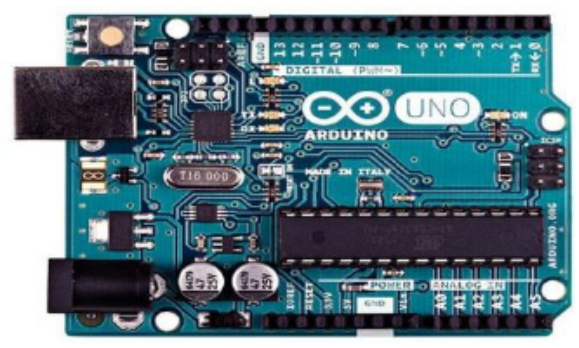

Figure 2 Arduino Uno Board

\subsection{Working Principle of Solar Panel}

Solar panels are devices that convert light into electricity (Figure 3). They are called "solar" panels because most of the time, the most powerful source of light available is the Sun, called Sol by astronomers. A solar panel is a collection of solar cells. When photons hit a solar cell, they knock electrons loose from their atoms. If conductors are attached to the positive and negative sides of a cell, it forms an electrical circuit. When electrons flow through such a circuit, they generate electricity. Multiple cells make up a solar panel, and multiple panels (modules) can be wired together to form a solar array. The more panels you can deploy, the more energy you can expect to generate. Photo Voltaic (PV) solar panels generate direct current (DC) electricity. With DC electricity, electrons flow in one direction around a circuit. The electrons move from the negative side of the battery, through the load, and return to the positive side of the battery. 


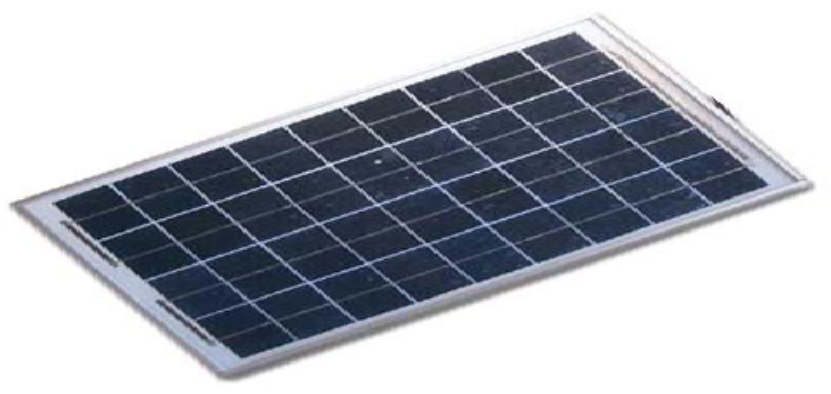

Figure 3 Solar Panel

\subsection{Interfacing of Real Time Clock (RTC)}

A real-time clock (RTC) is a computer clock (most often in the form of an integrated circuit) that keeps track of the current time. Although the term often refers to the devices in personal computers, servers and embedded systems, RTCs are present in almost any electronic device which needs to keep accurate time [4]. The DS1307 is a RTC chip (Figure 4).

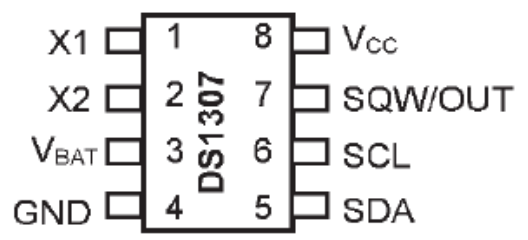

Figure 4 Pin Diagram of DS 1307 RTC chip

The following five pins are used: Vcc, GND, SCL, SDA, SQW.

$>5 \mathrm{~V}$ is given to $\mathrm{Vcc}$ pin to power on the RTC chip. If there is no $5 \mathrm{~V}$ signal, the chip goes to sleep using the coin cell for backup.

$>$ GND is connected to common power/data ground.

$>$ SCL pin is connected to the I2C clock SCL pin on the Arduino. On an Uno and, this is also known as A5.

SDA pin is connected to the I2C data SDA pin on the Arduino. On an Uno, this is also known as A4.

\subsection{Interfacing of Relay}

Relay is an electromagnetic device which is used to isolate two circuits electrically and connect them magnetically. They are often used to interface an electronic circuit (working at a low voltage) to an electrical circuit which works at very high voltage. For example, a relay can make a $5 \mathrm{~V}$ DC battery circuit to switch a $230 \mathrm{~V}$ AC mains circuit. Thus, a small sensor circuit can drive, say, a fan or an electric bulb.

A relay switch can be divided into two parts: input and output (Figure 5). The input section has a coil which generates magnetic field when a small voltage from an electronic circuit is applied to it. Commonly used relays are available in different configuration of operating voltages, viz. $6 \mathrm{~V}, 9 \mathrm{~V}, 12 \mathrm{~V}, 24 \mathrm{~V}$, etc. The output section consists of contactors which connect or disconnect mechanically. In a basic relay, there are three contactors: normally open (NO), normally closed (NC) and common (COM). At no input state, the COM is connected to NC. When the operating voltage is applied, the relay coil gets energized and the COM changes contact to NO. By using proper combination of contactors, electrical circuit can be switched on and off. 


\subsubsection{Pin diagram of a Relay module}

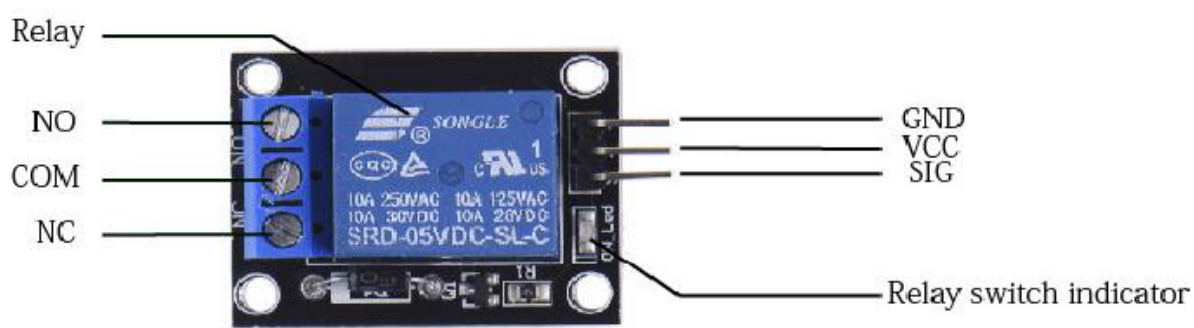

Figure 5 Pin Diagram of a Relay module

\subsubsection{Interfacing the relay modules to the Arduino}

Input:

It has a $1 \times 3(2.54 \mathrm{~mm}$ pitch) pin header for connecting power $(5 \mathrm{~V}$ and $0 \mathrm{~V})$, and for controlling the relay. The pins are marked on the PCB:

$>$ GND - Connect $0 \mathrm{~V}$ to this pin.

$>\mathrm{SIG}$ - Controls the relay, active low. Relay will turn on when this input goes below about 2.0V.

$>\mathrm{V}_{\mathrm{CC}}-\mathrm{Connect} 5 \mathrm{~V}$ to this pin. Used to power the opto couplers.

\section{Output:}

The 1 channel relay module could be considered like a series switches: 1 normally Open (NO), 1 normally closed (NC) and 1 common pins (COM).

$>\mathrm{COM}-$ Common pin.

$>\mathrm{NC}$ - Normally closed, in which case NC is connected with COM, when INT1 is set low and disconnected when INT1 is high.

NO - Normally Open, in which case NO is disconnected with COM1, when INT1 is set low and connected when INT1 is high.

\subsection{Mosquito Repeller}

Figure 6 shows a typical mosquito repeller system. The inner central layer is actually the hot mesh and is terminated as a high voltage source from the circuit enclosed in the lower handle area. The outer two layers are simply connected with the negative of the battery and constitute the ground for a return path to the central hot mesh line.

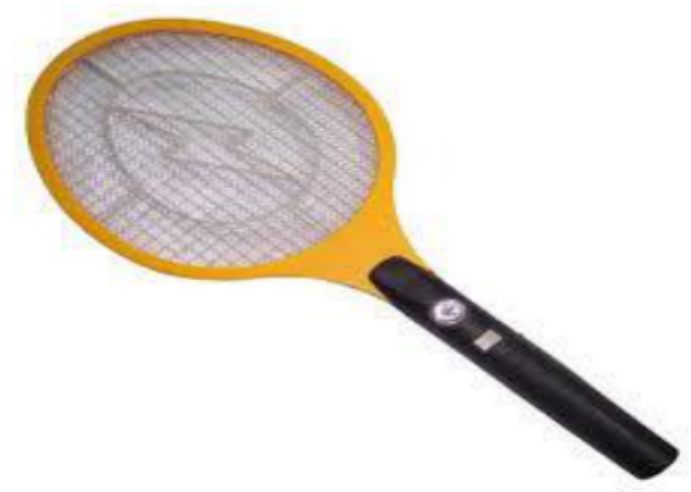

Figure 6 Mosquito Repeller with wire mesh 
However, since the inner layer and the outer layers are separated from each other at a reasonable distance, the high voltage from the central mesh is unable to arc-off through the outer layers. When a victim (a mosquito in this case) flies into this trap and bridges itself in between the layers, its body creates a low resistance path in between the layers of the mesh so that the high voltage is now able to arc through the body of the mosquito which is instantly electrocuted.

Figure 7 shows the schematic diagram of the repeller circuit [5]. This circuit supplies the required high voltages to the above mesh. The circuit is basically made up of three stages, viz. the power supply stage, the oscillator stage and the voltage booster stage. The power supply stage is a simple transformer less power supply circuit. The rectified voltage from the above power supply circuit is used to trickle charge an inbuilt rechargeable battery as long as the indoor electric mosquito zapper is not in use. When the battery gets fully charged, it just needs to be switched $\mathrm{ON}$ for the required function.

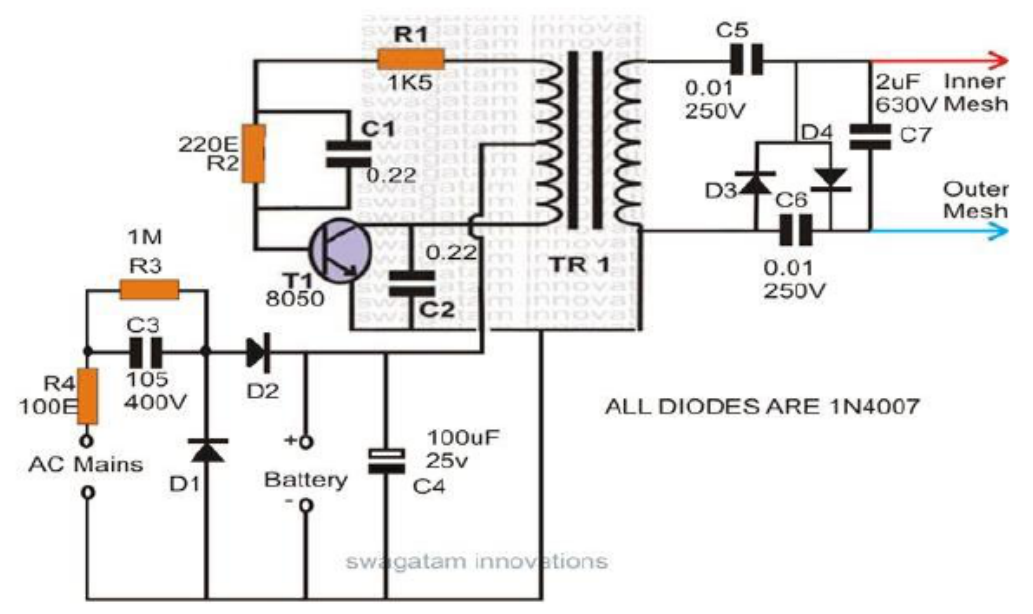

Figure 7 Schematic Diagram of a Mosquito Repeller circuit [5]

The switched-ON voltage from the battery is applied to the next oscillator stage comprising a transistor (T1) / transformer (Tr1) coupled oscillator configuration. The accompanied transformer steps-up the oscillating frequency at its secondary winding and functions like a smaller version of an inverter. The stepped-up AC at the output of the transformer is further boosted up through a diode/capacitor (D3, D4, C5, C6) ladder network. The boosted voltage ranging several hundred volts gets stored inside an output $\mathrm{AC}$ capacitor $\mathrm{C} 7$ across the output HOT ends which are finally terminated to the bat meshes and becomes ready to "shoot". The moment a mosquito or any bug comes in contact with the meshes, the stored high voltage in the capacitor discharges violently through the body of the entangled bug creating a big spark and electrocuting it instantly.

\subsection{Rechargeable SMF Battery}

A $12 \mathrm{~V}$ rechargeable sealed maintenance free (SMF) battery is used to power on the system. SMF batteries are designed to offer reliable, consistent and low maintenance power for various applications. These batteries can be subject to deep cycle applications and require minimum maintenance in rural and power deficit areas. 


\section{SOFTWARE TOOLS}

\subsection{Arduino IDE}

Arduino Integrated Development Environment (IDE) is the environment where we can write code, compile it and upload it to the Arduino board. IDE is basically a software that enables better and assisted code editing, compiling and debugging. The Arduino IDE runs on the Java platform. Arduino IDE has inbuilt functions and commands that though work on Java platform, are customized to run on the arduino development board. Thus, Arduino IDE serves for code editing, its compilation, debugging and then burning the code into the arduino board [6].

\section{SCHEMATIC DIAGRAM}

Figure 8 shows the complete block schematic diagram of the mosquito repeller system.

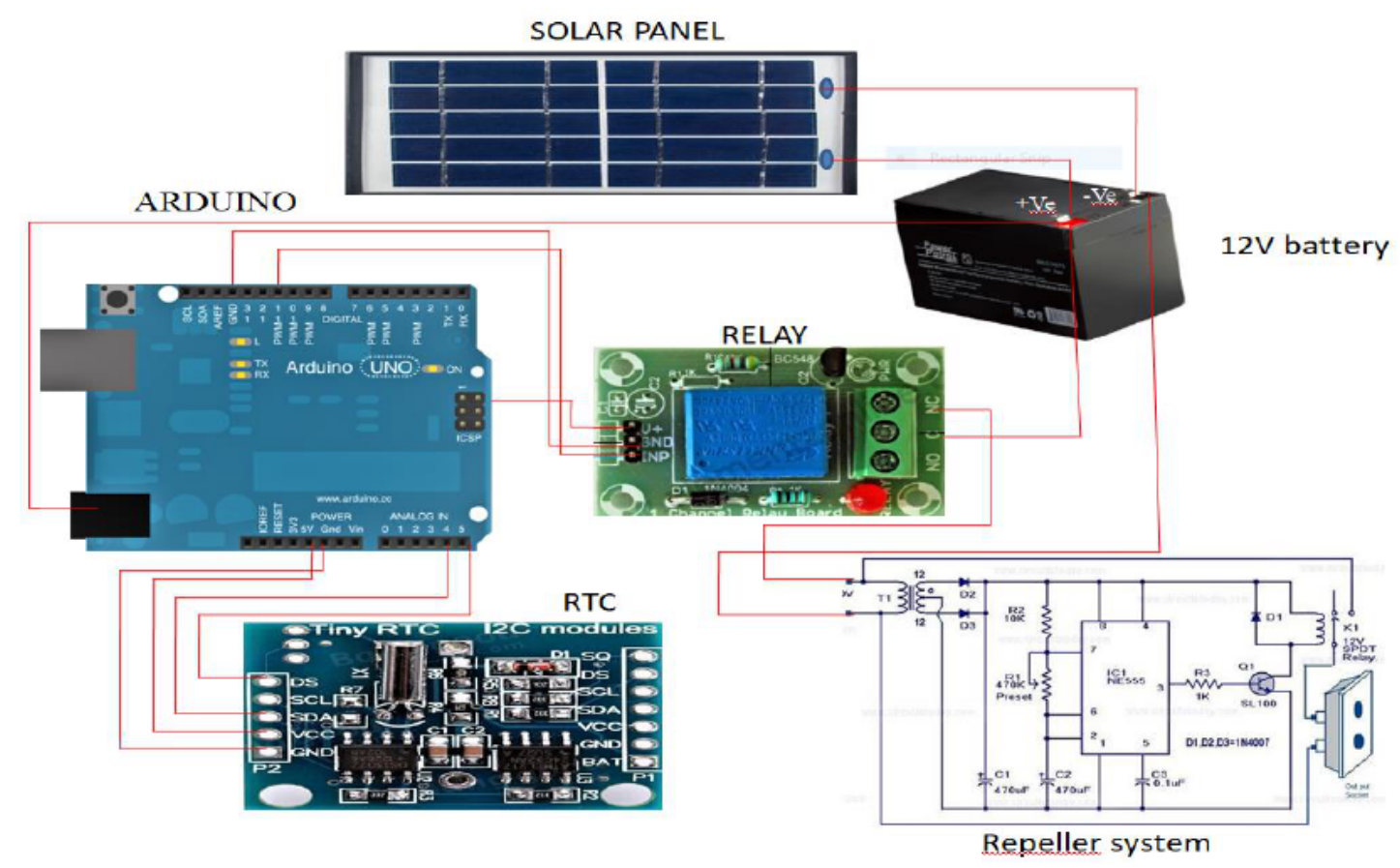

Figure 8 Complete Block Schematic Diagram of the Mosquito Repeller system

Here, A4 and A5 pins of Arduino board are connected to data pins, SDA and SCL of RTC. $\mathrm{V}_{\mathrm{CC}}$ of RTC is connected to $5 \mathrm{~V}$ of Arduino, and GND pin of RTC is grounded. The control pin of relay is connected to pin 11 of Arduino, $\mathrm{Vcc}$ of relay is connected to $5 \mathrm{~V}$ of Arduino, and the GND pin of relay is grounded. Relay NC is connected to one terminal of repeller system, whereas the COM is connected to positive terminal of battery. The negative terminal of battery is connected to other terminal of repeller system.

\section{FLOWCHART}

Figure 9 shows the flowchart of the sequence of events that occur in the mosquito repeller system. Observe that the repeller system is turned ON before $6 \mathrm{AM}$ and after $6 \mathrm{PM}$. 


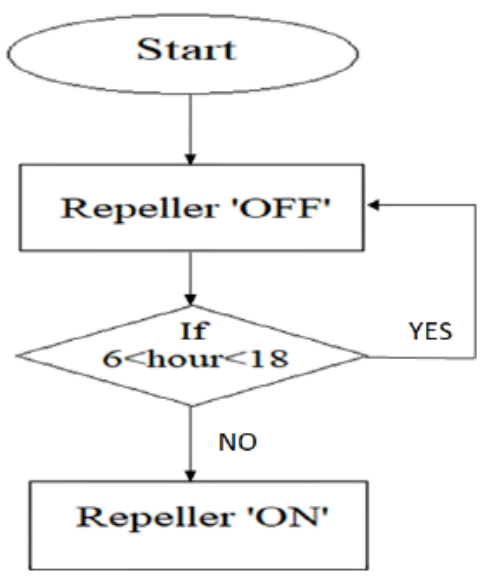

Figure 9 Flowchart of Mosquito Repeller system

\section{EXPERIMENTAL SETUP}

Figure 10 shows the experimental setup of the mosquito repeller system. The solar energy driven mosquito repeller system is intended to give jerk to mosquitoes. The moment a mosquito or any bug comes in contact with the meshes, the stored high voltage in the capacitor, discharges violently through the body of the entangled bug, creating a spark and electrocuting it instantly. This system uses solar panel as an energy source and a rechargeable battery as the energy storage element to store the harnessed energy. A relay is used to turn on the repeller and is connected to pin 11 of Arduino Uno. Voltage regulators are used in order to provide regulated output of $+5 \mathrm{~V}$ and $+9 \mathrm{~V}$ respectively. RTC is used in order to track the current time. The microcontroller of the Arduino Uno board is programmed in order to show the updated calendar values and also status of repeller system making the relay high or low.

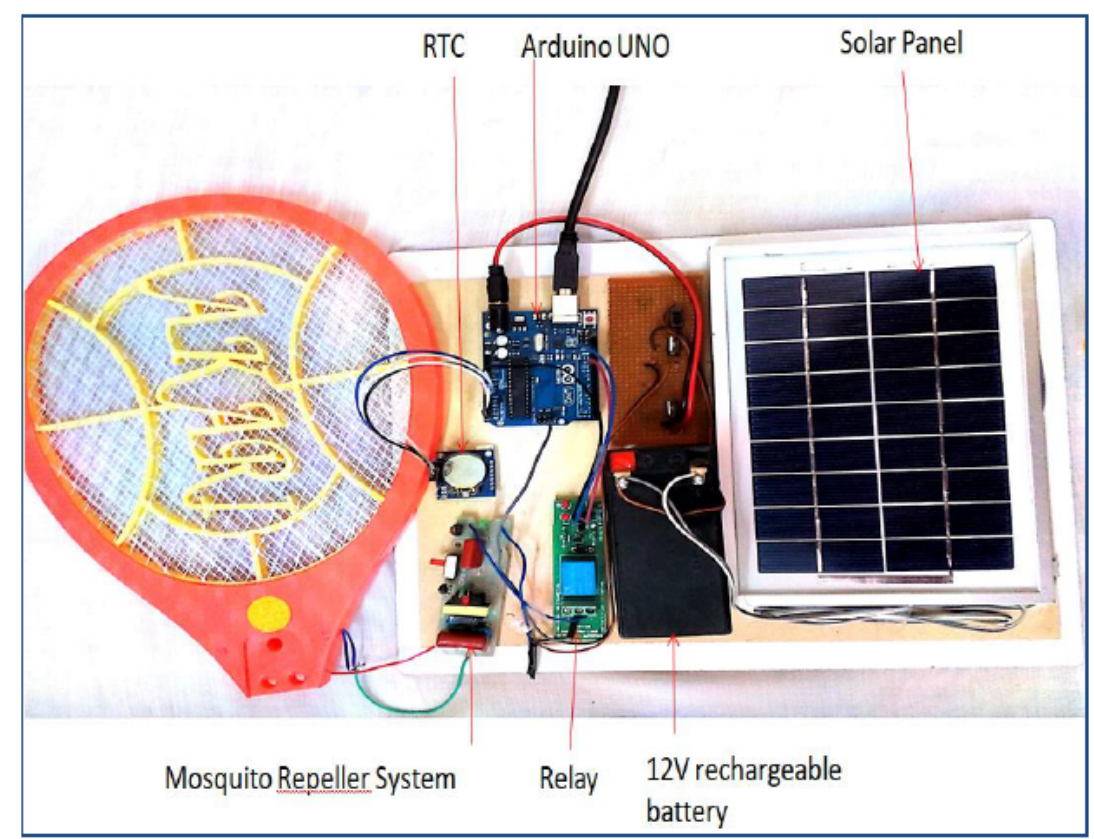

Figure 10 Experimental Setup of Mosquito Repeller system 


\section{EXPERIMENTAL RESULTS}

\subsection{When repeller system is "ON"}

Figure 11 shows the status in the serial monitor of Arduino when the mosquito repeller system is ON. Observe that the time stamp is greater than 6 PM.

\begin{tabular}{l}
\hline CoM18 (Arduino/Genuino Uno) \\
\hline Iime:22:49:41 \\
Date: $8 / 1 / 2019 /$ Mosquito Repeller 'On' \\
Time:22:49:41 \\
Date: $8 / 1 / 2019 /$ Mosquito Repeller 'ON' \\
Time:22:49:42 \\
Date: $8 / 1 / 2019 /$ Mosquito Repeller 'ON' \\
Iime:22:49:43 \\
Date: $8 / 1 / 2019 /$ Mosquito Repeller 'ON' \\
\hline
\end{tabular}

Figure 11 Status of Mosquito Repeller system displayed in serial monitor as 'ON'

Figure 12 shows the experimental results when repeller system is 'ON'. Observe that relay output is ' 0 ', when repeller is ' $\mathrm{ON}$. To test the system, a small tester is brought in contact with the wire mesh. At this point of time, a spark is observed.
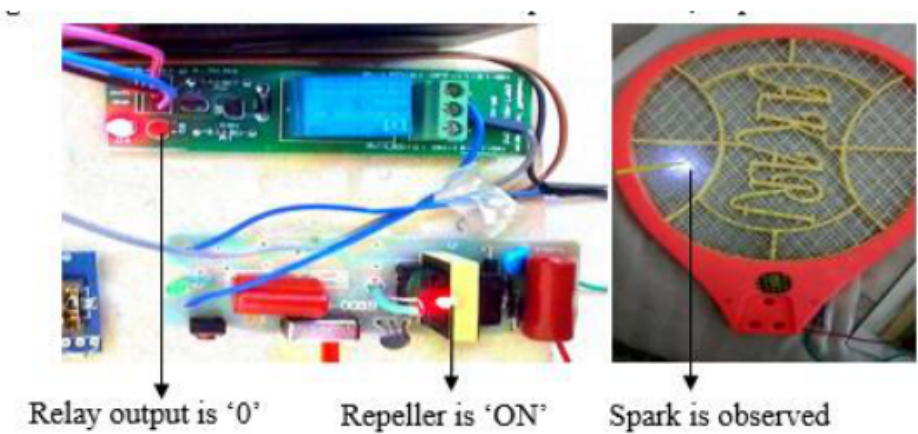

Figure 12 Experimental Results when Mosquito Repeller system is ' $\mathrm{ON}$ '

7.2. When repeller system is "OFF"

Figure 13 shows the status in the serial monitor of Arduino when the mosquito repeller system is OFF. Observe that the time stamp is earlier to $6 \mathrm{PM}$.

\begin{tabular}{|l|}
\hline \\
\hline Time:12:47:46 COM18 (Arduino/Genuino Uno) \\
Date: $8 / 1 / 2019 /$ Mosquito Repeller 'OFF' \\
Time:12:47:47 \\
Date: $8 / 1 / 2019 /$ Mosquito Repeller 'OFF' \\
\hline
\end{tabular}

Figure 13 Status of Mosquito Repeller system displayed in serial monitor as 'OFF'

Figure 14 shows the experimental results when the repeller system is 'OFF'. Observe that relay output is ' 1 ', when repeller is 'OFF'. To test the system, a small tester is brought in contact with the wire mesh. At this point of time, no spark is observed as repeller system is set to OFF between $6 \mathrm{AM}$ and $6 \mathrm{PM}$. 


\section{Dr. Dhiraj Sunehra}

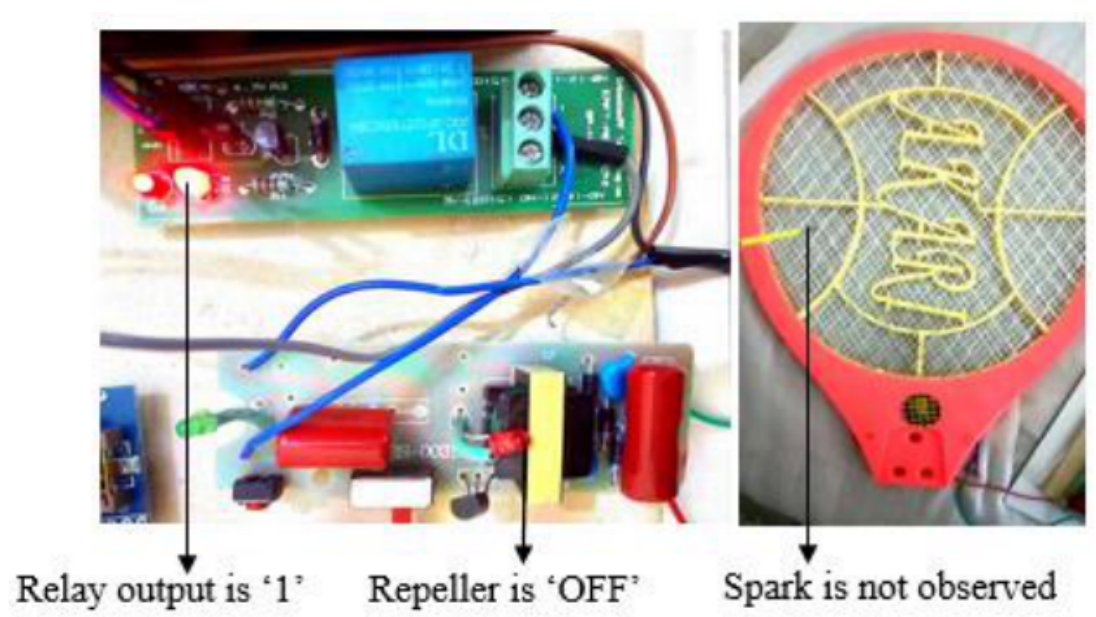

Figure 14 Experimental Results when Mosquito Repeller system is 'OFF'

\section{CONCLUSIONS}

In this paper, a solar energy driven mosquito repeller system has been implemented. This system can effectively replace the chemical repellents which affect the environment. The designed system consumes very less power and can be effectively used in lawns and environment parks to repel the mosquitoes. As a future work, this mosquito repeller system can be replaced by a highly efficient high power repeller, so that it can cover more area and can be used to repel insects from farms.

\section{REFERENCES}

[1] https://www.nationalgeographic.com

[2] https://www.studentenergy.org

[3] https://www.arduino.cc

[4] https://www.sparkfun.com/datasheets/Components/DS1307.pdf

[5] https://www.brighthubengineering.com

[6] https://www.arduino.cc/en/guide/environment

[7] Sajid M. Sheikh and Tshoganyetso Mangadi, An Arduino Based Building Lights Management System, International Journal of Electrical Engineering and Technology (IJEET), Volume 4, Issue 6, November - December (2013), pp. 45-59

[8] Mohammad Mohiddin, Musipatla Premalatha, Bodapally Kedarnath, K. Sanath Kumar and K. Venkata Krishna Prasad. Implementation of Brain - Computer Interface Technology using Arduino. International Journal of Electrical Engineering \& Technology, 8(2), 2017, pp. 25-35

[9] K. Anusha, L. Baala Gajakreedan, S. Nishanth Kumar and S. Vignesh Rajarathinam, Persistence of Vision using Arduino. International Journal of Electronics and Communication Engineering and Technology, 8(4), 2017, pp. 7-12.

[10] Anis Kazi, Sourabh Arbune, N. Ruban and V. Bagyaveereswaran, Position Control of Visual Prosthetic System Using Arduino Processor, International Journal of Mechanical Engineering and Technology, 9(9), 2018, pp. 1131-1139

[11] T. Sridevi, Smart Wireless Sensor Network for Agricultural Environment Using Arduino UNO. International Journal of Electronics and Communication Engineering and Technology, 9(4), 2018, pp.10-21. 